\title{
eJRIEPS
}

Ejournal de la recherche sur l'intervention en éducation physique et sport

Hors-série $N^{\circ} 4$ | 2021

Actes de la $11^{\mathrm{e}}$ Biennale de l'ARIS, Liège, 25-28 février 2020

\section{Cadre d'analyse des facteurs qui caractérisent une perception positive de l'activité physique chez les élèves du primaire}

A Framework for Analyzing the Factors that Characterize Positive Perceptions of Physical Activity in Elementary School Students

Jolaine Désautels, Sylvie Beaudoin et Sylvain Turcotte

\section{OpenEdition \\ Journals}

Édition électronique

URL : https://journals.openedition.org/ejrieps/6375

DOI : $10.4000 /$ ejrieps. 6375

ISSN : 2105-0821

Éditeur

ELLIADD

\section{Référence électronique}

Jolaine Désautels, Sylvie Beaudoin et Sylvain Turcotte, « Cadre d'analyse des facteurs qui

caractérisent une perception positive de l'activité physique chez les élèves du primaire », eJRIEPS [En ligne], Hors-série $N^{\circ} 4$ | 2021, mis en ligne le 10 juin 2021, consulté le 03 juillet 2021. URL : http:// journals.openedition.org/ejrieps/6375; DOI : https://doi.org/10.4000/ejrieps.6375

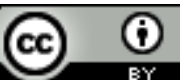

La revue eJRIEPS est mise à disposition selon les termes de la Creative Commons Attribution 4.0 International License. 


\section{Cadre d'analyse des facteurs qui caractérisent une perception positive de l'activité physique chez les élèves du primaire}

Jolaine Désautels*, Sylvie Beaudoin*, Sylvain Turcotte*

*Faculté des sciences de l'activité physique, Université de Sherbrooke, Québec.

Résumé : Les interventions en milieu scolaire doivent favoriser le développement d'une perception positive (PP) de l'activité physique (AP) chez les élèves pour avoir des répercussions à long terme sur leur mode de vie actif. Toutefois, les perceptions des élèves en lien avec l'AP sont sous-représentées dans la littérature scientifique et plus particulièrement lors du déploiement des interventions en milieu scolaire primaire. Cet article a donc pour but de présenter : (1) les facteurs qui caractérisent une PP de la pratique d'AP chez les élèves du milieu scolaire primaire et (2) de modéliser un cadre d'analyse permettant de catégoriser ces facteurs. Une recherche documentaire a été menée afin d'identifier les facteurs qui caractérisent une PP de l'AP chez les élèves du primaire en plus d'élaborer un cadre d'analyse. Les résultats mettent en évidence 24 facteurs caractérisant une PP de l'AP chez les élèves $d u$ primaire. Ces facteurs sont présentés dans un cadre d'analyse élaboré à partir du modèle socio-écologique de McLeroy et al. (1988). Ce cadre d'analyse est pertinent pour identifier, décrire et caractériser les perceptions des élèves dans le contexte où des stratégies d'intervention visant à augmenter leur niveau d'AP sont mises en place en milieu scolaire. Mots clés: modèle socio-écologique; perception positive ; activité physique; élèves du primaire.

A Framework for Analyzing the Factors that Characterize Positive Perceptions of Physical Activity in Elementary School Students

Summary: Interventions must promote the development of a positive perception (PP) about physical activity (PA) to have long-term effects on students' active lifestyle. However, their perceptions of PA are under-represented in the scientific literature and more particularly during the deployment of interventions in primary school. The purpose of the present paper was to present: (1) the factors that characterize a PP of PA practice in elementary school students and (2) to model an analytical framework to categorize these factors. A review of the literature was 
eJRIEPS Hors Série 4 Juillet 2021

conducted to identify the factors that characterize a PP of PA in elementary school students in addition to developing a framework for analysis. The results highlight twenty-four factors characterizing a PP of PA in elementary school students. These factors are presented in an analytical framework developed on the basis of the socio-ecological model of McLeroy et al. (1988). This analytical framework is relevant for identify, describe, and characterize students' perceptions in the context where intervention strategies aimed at increasing their level of PA are implemented in schools.

Key words: ecological framework; positive perception; physical activity; elementary school students.

\section{Problématique}

Compte tenu du fort lien qui existe entre l'activité physique (AP) et la santé des jeunes (ParticipACTION, 2020), le milieu scolaire est identifié comme un endroit privilégié pour mettre en place des interventions visant à promouvoir le mode de vie actif chez les jeunes. Bien que plusieurs interventions en lien avec l'AP soient mises en œuvre dans les écoles, il semble qu'elles n'aient pas toujours l'effet escompté, soit de promouvoir des attitudes et des valeurs en lien avec l'AP susceptibles d'inciter les élèves à adopter un mode de vie actif à long terme. Cet article porte donc sur l'identification des facteurs qui contribuent au développement d'une PP de l'AP chez les élèves afin qu'ils intègrent l'AP à leur mode de vie.

\subsection{Portrait de la situation de la pratique d'activité physique}

Le nombre d'heures que les jeunes consacrent à la pratique d'activités sédentaires durant leurs loisirs ne cesse d'augmenter (Carson et al., 2016). Ces comportements récréatifs sédentaires entraînent des risques importants menant au développement de maladies dites « non transmissibles " telles que l'obésité, le diabète, l'hypertension et le risque de dépression (Organisation mondiale de la santé [OMS], 2010). Ces loisirs sédentaires, qui se caractérisent par une très faible dépense énergétique, sont notamment associés aux activités passées devant des écrans tels que l'ordinateur, les jeux vidéo ou la télévision (Institut de la statistique du Québec, 2015 ; ParticipACTION, 2020). 
eJRIEPS Hors Série 4 Juillet 2021

En plus de l'augmentation préoccupante du nombre d'heures accordées aux activités sédentaires, il semble que les jeunes soient trop peu actifs et que leur condition physique se soit détériorée au cours des dernières décennies. Ainsi, selon une étude réalisée auprès de 1,6 millions de jeunes de 11 à 17 ans répartis dans 146 pays, 81\% d'entre eux ne rencontrent pas les directives d'AP recommandées, soit un minimum de 60 minutes par jour d'AP d'intensité modérée à élevée comprenant une variété d'activités aérobies et d'activités pour renforcer les muscles et les os (Guthold, Stevens, Riley \& Bull, 2020 ; OMS, 2010). Selon le bulletin 2020 de ParticipACTION, seulement $39 \%$ des jeunes Canadiens âgés de 5 à 17 ans atteignent ce volume d'AP. La Société canadienne de physiologie de l'exercice (2016) recommande en plus d'ajouter quotidiennement plusieurs heures d'une variété d'AP d'intensité légère structurées et non structurées.

Des actions doivent donc être mises en œuvre pour augmenter le niveau d'AP des jeunes et ce, partout dans le monde (Guthold et al., 2020).

\subsection{Bienfaits associés à la pratique d'activité physique}

Bien que toutes les modalités d'AP soient associées à une meilleure santé, c'est l'AP d'intensité moyenne à élevée qui offre le plus de bienfaits (Saunders et al., 2016). Chez les jeunes de 5 à 17 ans, ces bienfaits sont nombreux et reliés aux dimensions physique, psychologique, sociale et cognitive de la santé (Comité scientifique de Kino-Québec, 2011 ; Poitras et al. 2016). De nombreuses études montrent, entre autres, que des niveaux élevés d'AP chez les jeunes sont associés à une meilleure capacité cardiovasculaire et santé osseuse (Poitras et al., 2016), à une plus grande estime de soi (Guddal et al., 2019), à une probabilité plus faible de présenter des symptômes de dépression (Eddolls et al., 2018), à des comportements sociaux plus adéquats (Moeijes, Van Busschbach, Bosscher \& Twisk, 2018), à la diminution de l'inattention et de l'hyperactivité (Pontifex, Parks, Henning \& Kamijo, 2015) et à de meilleures performances scolaires (Chu, Chen, Pontifex, Sun \& Chang, 2019). La pratique d'AP fait donc partie intégrante d'un mode de vie sain et elle est essentielle pour la santé des jeunes.

\subsection{Activité physique en contexte scolaire}

Il semble que la pratique d'AP à l'école constitue un des moyens les plus efficaces pour améliorer les différentes dimensions de la santé des jeunes (Coalition québécoise sur la problématique du poids, 2013 ; OMS, 2019). Le milieu scolaire est d'ailleurs reconnu comme 


\section{eJRIEPS Hors Série 4 Juillet 2021}

un contexte idéal pour contribuer à augmenter le niveau d'AP des enfants et des adolescents, notamment grâce à la possibilité d'intervenir auprès de l'ensemble des élèves (Pate et al., 2006). Puisque les jeunes y passent une grande partie de leur vie, le milieu scolaire constitue un contexte favorable pour acquérir les connaissances ainsi que pour développer les compétences permettant de faire des choix favorables à la santé et davantage d'AP (OMS, 2019). D'ailleurs, selon Gadais (2015), les stratégies d'intervention qui sont initiées en milieu scolaire pour augmenter le niveau d'AP des jeunes sont parmi celles qui ont le plus d'impact sur leur mode de vie actif compte tenu de l'encadrement qu'ils reçoivent de la part des enseignants et de la structure qui favorise une meilleure participation des jeunes.

Au Québec, pour favoriser l'engagement des jeunes dans un mode de vie actif, la mesure gouvernementale 15023 - À l'école, on bouge! - a été mise en place dans les établissements d'éducation et d'enseignement préscolaire et primaire. Implantée au début de l'année scolaire 2017 dans les écoles, cette mesure a pour objectif de rendre les élèves physiquement actifs tous les jours de classe pendant au moins 60 minutes, les cours d'éducation physique et à la santé (EPS) prévus à l'horaire des élèves étant inclus dans le volume d'AP à atteindre (Gouvernement du Québec, 2018). Les écoles qui bénéficient de cette mesure reçoivent une aide financière régressive pour une durée de trois ans. Son objectif est de soutenir les équipesécoles pour qu'elles s'engagent dans un changement de pratiques, tant dans l'organisation que dans les interventions, afin que l'intégration quotidienne de 60 minutes d'AP soit maintenue audelà de la durée de cette aide financière (Gouvernement du Québec, 2018). Les écoles bénéficiaires sont responsables d'identifier les moyens qu'elles souhaitent mettre en place afin de bonifier les occasions pour rendre les élèves physiquement actifs pendant la journée, et ce, en fonction du contexte dans lequel elles se trouvent et des besoins des élèves. Cette mesure gouvernementale doit être implantée dans toutes les écoles primaires du Québec d'ici l'année scolaire 2027-2028 (Gouvernement du Québec, 2018).

\subsection{Limites des interventions à l'école}

Les mesures et stratégies d'intervention visant à rendre les jeunes physiquement actifs en milieu scolaire n'auraient parfois que des effets à court terme sur leur mode de vie (Dobbins Husson, DeCorby \& LaRocca, 2013 ; Metcalf, Henley \& Wilkin, 2012).

Certaines barrières semblent nuire à leur mise en place et, par le fait même, limitent l'effet escompté chez les jeunes, soit l'adoption d'un mode de vie actif (Harvey, Pearson, Sanzo \& 


\section{eJRIEPS Hors Série 4 Juillet 2021}

Lennon, 2017). Parmi ces barrières on retrouve notamment: (1) la difficulté d'accès aux installations et aux équipes sportives (Bauer, Yang \& Austin, 2004; Carlin, Murphy \& Gallagher, 2015; Harvey et al., 2017 ; James et al., 2018; Van den Berg, Vos, De Groot, Singh \& Chinapaw, 2018) ; (2) les interactions difficiles avec les pairs (Bauer et al., 2004 ; Carlin et al., 2015; Harvey et al., 2017 ; Van den Berg et al., 2018) ; (3) les priorités des élèves qui comblent une majeure partie de leur agenda (Bauer et al., 2004 ; Carlin et al., 2015 ; Harvey et al., 2017) ; (4) l'implication variable des membres de l'équipe-école dans la mise en place des initiatives en lien avec l'AP à l'école ; (5) le sentiment de non-compétence des élèves (Carlin et al., 2015; Harvey et al., 2017 ; Van den Berg et al., 2018) ; (6) les politiques trop strictes de l'école à propos de la sécurité des élèves (Bauer et al., 2004 ; Harvey et al., 2017 ; Van den Berg et al., 2018) ; et (7) l'incompatibilité des AP proposées avec les intérêts des élèves (Carlin et al., 2015; Harvey et al., 2017 ; James et al., 2018 ; Van den Berg et al. 2018).

Bien que les barrières à la pratique d'AP des élèves soient bien documentées, il semble qu'elles soient rarement considérées dans la mise en place des interventions en lien avec l'AP en milieu scolaire (Brunton, Harden, Rees, Kavanagh, Oliver \& Oakley, 2003 ; Harvey et al., 2017). En effet, les décideurs politiques et les intervenants scolaires, qui jouent un rôle essentiel dans la conception et la mise en place d'interventions en lien avec l'AP en milieu scolaire, approchent souvent la situation selon leur propre perspective en croyant que les élèves apprécieront les interventions proposées (Hyndman, 2016). James et al. (2018) ajoutent que ces acteurs reçoivent souvent peu d'informations et de commentaires de la part des bénéficiaires clés, soit les élèves. Pourtant, ces derniers expriment qu'ils veulent être consultés dans la mise en place des interventions qui les incitent à adopter un mode de vie actif (Harvey et al., 2017) et que leurs idées soient considérées (Van den Berg et al., 2018). Sans cela, on laisse croire aux élèves qu'ils ont peu d'influence dans les décisions qui les concernent. En conséquence, un écart se crée entre les interventions réalisées et les réels besoins des élèves en ce qui concerne l'AP en milieu scolaire (Hyndman, 2016; James et al., 2018). Cet écart pourrait être responsable du développement d'une perception négative de l'AP durant l'enfance et l'adolescence et nuire à l'engagement des jeunes dans la pratique d'AP (Cardinal, Yan \& Cardinal, 2013; Hayes, 2017; Van den Berg et al., 2018).

\subsection{Favoriser l'engagement des jeunes à long terme dans la pratique d'activité physique}




\section{eJRIEPS Hors Série 4 Juillet 2021}

Pour favoriser l'engagement des élèves dans la pratique d'AP et pour assurer le succès à long terme des interventions qui visent à les rendre physiquement actifs, Jacquez et al. (2013) proposent de les impliquer dans l'élaboration des AP proposées à l'école. La possibilité de faire des choix ainsi que la perception d'être autonome dans sa pratique d'AP seraient associées à une expérience plus positive chez les élèves en plus d'améliorer leur sentiment d'efficacité personnelle (Cairney, Kwan, Velduizen, Hay, Bray \& Fought, 2012 ; Hayes, 2017). Cela exercerait un effet positif sur leur engagement à long terme dans la pratique d'AP et sur leur perception quant à l'AP (Harvey et al., 2017).

En plus d'avoir une influence considérable sur le mode de vie actif, il semble que les jeunes soient plus susceptibles d'intégrer l'AP à leur quotidien lorsqu'ils la perçoivent positivement (Burns, Fu \& Podlog, 2017 ; Hayes, 2017). Les interventions qui sont faites en milieu scolaire devraient donc favoriser le développement d'une PP de l'AP pour avoir des répercussions à long terme sur le mode de vie actif des jeunes (Burns et al., 2017 ; Cairney et al., 2012). Une PP de l'AP se définit comme étant un point de vue ou un état d'esprit favorable quant à l'AP qui incite l'élève à s'engager dans une pratique d'AP et qui lui permet d'en retirer une satisfaction personnelle (Grenier, 2006 ; Legendre, 2005).

\subsection{Perception des élèves quant à l'activité physique}

Jusqu'à présent, les perceptions des élèves sont sous-représentées dans la littérature scientifique (Harvey et al., 2017), notamment en ce qui a trait aux facteurs qui favorisent la PP de I'AP chez les jeunes d'âge primaire (Cairney et al., 2012 ; Carlin et al., 2015 ; Everley \& Macfadyen, 2017 ; Hayes, 2017). Une meilleure connaissance de ces facteurs lors du déploiement des interventions en milieu scolaire pourrait fournir des informations importantes pour élaborer et améliorer les interventions qui visent à rendre les élèves physiquement actifs en milieu scolaire (Harvey et al., 2017 ; Van den Berg et al., 2018).

Les constats précédents nous ont donc amené à réaliser une recherche et une analyse documentaire afin de répondre à la question suivante : quels sont les facteurs favorisant une PP de l'AP chez les élèves du primaire?

\section{Cadre théorique}

L'approche utilisée pour réaliser l'analyse documentaire repose sur les travaux émanant de l'approche socio-écologique du développement humain (Bronfenbrenner, 1977). Cette 


\section{eJRIEPS Hors Série 4 Juillet 2021}

approche a permis de mieux comprendre comment s'articulent, autour de l'élève, les facteurs qui influencent sa perception de l'AP. Cette section présente donc les fondements théoriques de l'approche socio-écologique ainsi que le cadre théorique retenu, soit le modèle socioécologique de McLeroy, Bibeau, Steckler et Glanz (1988).

\subsection{Approche socio-écologique}

L'approche socio-écologique est présentée, notamment, comme un cadre utile pour la recherche et les interventions en lien avec l'activité physique (Sallis, Owen \& Fisher, 2008). Cette approche repose sur une notion fondamentale: celle de l'interaction et de l'interdépendance entre l'individu et les déterminants sociaux et environnementaux (Brofenbrenner, 1977). L'approche socio-écologique désigne donc une façon d'aborder les problématiques selon une démarche qui englobe les individus et tous les déterminants qui les entourent afin de favoriser l'adoption ou la transformation des comportements individuels (Vors et al., 2020). D'ailleurs, les interventions qui agissent sur plusieurs déterminants à la fois sont plus efficaces pour engendrer un changement de comportement (Sallis et al., 2008). Les modèles écologiques, quant à eux, schématisent les relations entre les déterminants individuels, sociaux et environnementaux. Ainsi, les interactions ne sont pas seulement présentes entre l'individu et un seul déterminant social ou environnemental, mais entre la personne et plusieurs niveaux à la fois (Bouchard, 1987). Enfin, les modèles écologiques fournissent un cadre de réflexion et d'analyse des programmes et des actions de prévention et de promotion de la santé (Richard, Barthélémy, Tremblay, Pin \& Gauvin, 2013).

Les perceptions des élèves quant à l'AP sont influencées par une multitude de facteurs qui se déploient dans l'environnement de l'élève et qui semblent avoir une influence l'un sur l'autre (Eberline, Judge, Walsh \& Hensley, 2018 ; Devís-Devís, Beltrán-Carrillo \& Peiró-Velert, 2015). Ainsi, l'utilisation d'un modèle socio-écologique permettra catégoriser ces facteurs et de mettre en perspective les liens qui les unissent.

\subsection{Modèle socio-écologique de McLeroy et al. (1988)}

Le modèle socio-écologique de McLeroy et al. (1988), est le cadre théorique utilisé pour réaliser l'analyse documentaire et pour élaborer le cadre d'analyse. Ce modèle, présenté dans la figure 1, est organisé en cercles concentriques et est composé des cinq niveaux suivants: (1) intrapersonnel ; (2) interpersonnel ; (3) institutionnel ; (4) communautaire ; et (5) politique. Le 


\section{eJRIEPS Hors Série 4 Juillet 2021}

niveau intrapersonnel est caractérisé par les croyances, les capacités, les comportements ou même les connaissances de l'individu, entre autres. Le deuxième niveau, c'est-à-dire le niveau interpersonnel, se rapporte entre autres à la famille, aux amis et aux collègues de travail. Le troisième niveau, soit les facteurs institutionnels, est représenté par les institutions sociales et leurs caractéristiques organisationnelles. On y retrouve notamment le milieu scolaire et les intervenants qui y travaillent, dont l'enseignant en EPS. Le quatrième niveau réfère aux relations informelles qu'un individu pourrait avoir avec différentes organisations ou institutions présentes dans sa communauté. Le dernier niveau, soit celui de la politique, réfère aux lois et aux politiques locales, de l'état et du pays (McLeroy et al., 1988).

Le modèle de McLeroy et al. (1988) a été retenu par rapport aux autres modèles socioécologiques puisqu'il laisse une place centrale à l'individu. Puisque cette étude s'intéresse exclusivement aux perceptions des élèves, il est pertinent que ces derniers occupent cette place significative dans le modèle. Ce modèle est aussi pertinent pour son aspect inductif qui offre la possibilité de former plusieurs catégories de facteurs pouvant être mis de l'avant par les élèves. 


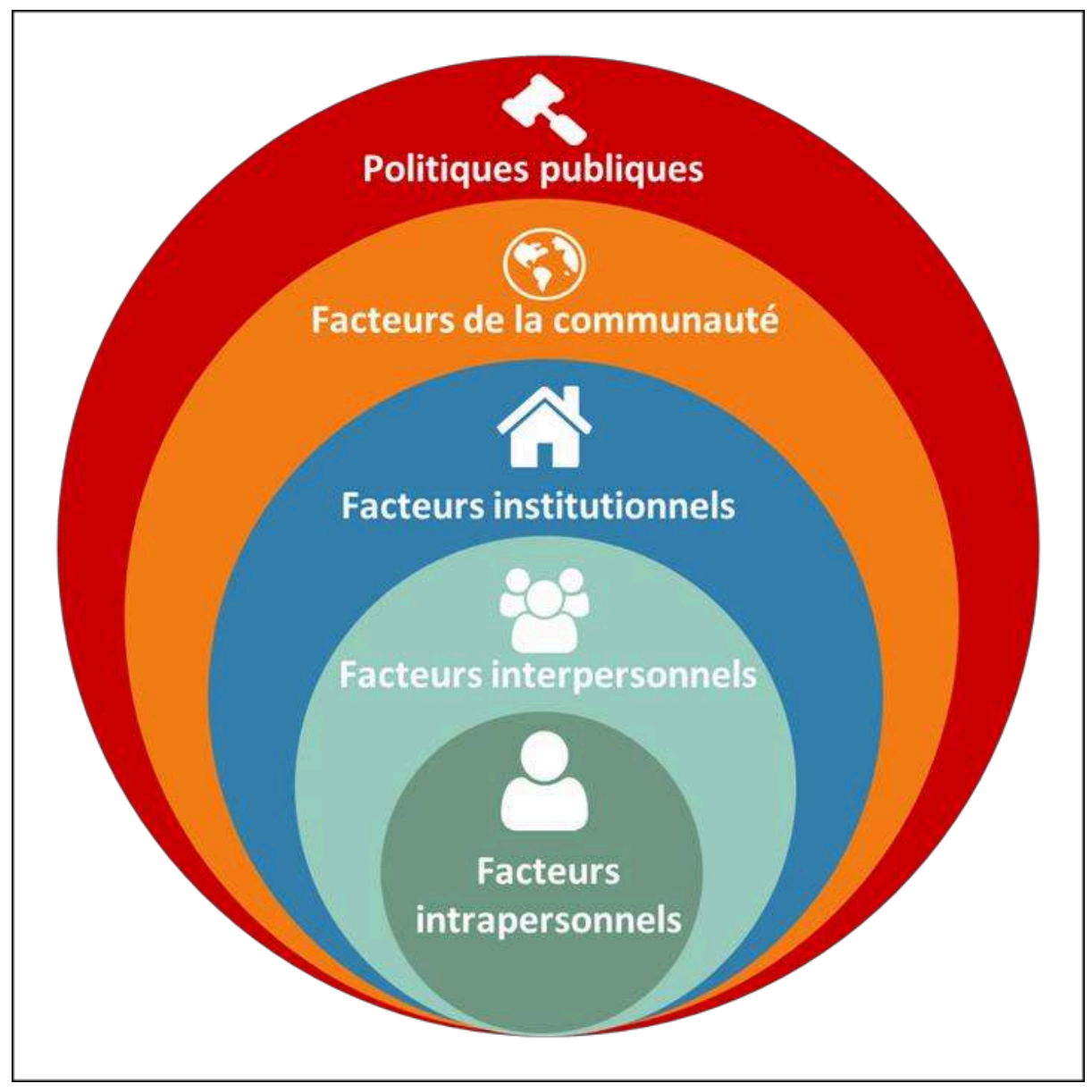

Figure 1. Modèle socio-écologique retenu (adapté de McLeroy et al.,1988)

\subsection{Objectifs}

Les objectifs de l'article sont (1) d'identifier les facteurs favorisant une PP de l'AP chez les élèves du primaire et (2) de modéliser un cadre d'analyse, à l'aide du modèle socio-écologique de McLeroy et al. (1988), permettant de catégoriser les facteurs favorisant une PP de l'AP.

\section{Méthodologie}

Afin d'identifier les facteurs qui caractérisent une PP de la pratique d'AP chez les élèves en milieu scolaire primaire, une recherche et une analyse documentaire ont été réalisées. Ensuite, une stratégie de modélisation à partir du modèle socio-écologique de McLeroy et al. (1988) a été mise en place pour élaborer le cadre d'analyse. Les sections suivantes présentent donc les étapes de la recherche et de l'analyse documentaire ainsi que la stratégie de modélisation. 


\section{eJRIEPS Hors Série 4 Juillet 2021}

\section{1. Étapes de la recherche documentaire}

La recherche documentaire, s'étant réalisée en novembre 2018, s'est déroulée en six étapes : (1) identification des concepts ; (2) recherche de mots clés ; (3) traduction des mots clés en langue anglaise ; (4) recherche avancée dans différentes banques de données ; (5) sélection des ressources documentaires pertinentes; et (6) recherche secondaire à partir de la bibliographie des ressources documentaires pertinentes. Ces étapes sont décrites plus en détail dans le paragraphe suivant et sont illustrées à la figure 2.

La première étape de la recherche documentaire a consisté à diviser la question de recherche afin de déterminer les différents concepts qui y sont présents. La question de recherche se divise en trois concepts distincts : (1) perception positive ; (2) activité physique ; et (3) élèves du primaire. Ensuite, des mots clés représentant les différents concepts de la question de recherche ont été identifiés. Ces derniers sont inscrits dans la figure 2. La quatrième étape a consisté à effectuer la recherche avancée dans les banques de données suivantes: ERIC, SPORTDiscus, MEDLINE, PubMed et Google Scholar. La majorité des ressources documentaires ont été repérées à partir de la banque de données SPORTDiscus. C'est pour cette raison qu'à la quatrième étape, présentée dans la figure 2, la recherche avancée est illustrée seulement pour la banque de données SPORTDiscus. Pour sélectionner les ressources documentaires pertinentes, le titre a d'abord été lu. Lorsqu'il correspondait à l'objet de recherche, le résumé était ensuite analysé. Pour conserver ou éliminer les ressources documentaires repérées dans les banques de données, différents critères d'inclusion et d'exclusion, présentés à l'étape 5 de la figure 2 , ont été déterminés. Toutefois, très peu d'études ont investigué les facteurs qui favorisent le développement d'une PP de l'AP chez les élèves du primaire, c'est pourquoi les critères de sélection qui ont été établis pour la recherche documentaire ont été élargis. Ainsi, des ressources documentaires qui incluaient une population plus âgée que celle ciblée au départ par la question de recherche, soit les élèves du primaire, ont aussi été conservées. Les articles retenus exposent donc des facteurs favorisant une PP de l'AP chez des élèves de 5 à 17 ans. Au départ, les ressources documentaires ayant une date de publication entre 2009 et 2020 étaient privilégiées. Toutefois, certaines d'entre elles ayant une date antérieure ont été conservées puisqu'elles étaient pertinentes par rapport au sujet de la recherche. Les ressources documentaires qui n'étaient pas en lien avec l'AP, qui ne représentaient pas les perceptions des élèves ou qui étaient indisponibles en anglais ou en français n'étaient pas retenues. Enfin, une recherche 
eJRIEPS Hors Série 4 Juillet 2021

documentaire secondaire a été réalisée à partir des bibliographies des articles retenus à l'étape de la recherche avancée dans les banques de données. La sélection de ces articles s'est réalisée avec les mêmes critères d'inclusion et d'exclusion précédemment décrits. Au total, l'exercice a permis d'identifier 19 articles qui ont été retenus pour l'analyse documentaire. 


\section{Étape 1 :}

Division des concepts de l'objectif de recherche \#1

Identifier les facteurs qui caractérisent une perception positive de l'activité physique chez les élèves du primaire

$\checkmark$

\begin{tabular}{|c|c|c|c|}
\hline \multicolumn{4}{|c|}{$\begin{array}{c}\text { Étape } 2 \text { et } 3 \text { : } \\
\text { Recherche de mots clés et traduction en anglais }\end{array}$} \\
\hline Concepts & Perception positive & Activité physique & Élèves du primaire \\
\hline Français & $\begin{array}{l}\text { - Perception favorable } \\
\text { - Attitude positive ou favorable } \\
\text { - Opinion positive ou favorable } \\
\text { - Plaisir } \\
\text { - Point de vue } \\
\text { - Expérience }\end{array}$ & $\begin{array}{l}\text { - Sport } \\
\text { - Exercice } \\
\text { - Éducation physique } \\
\text { - Programmes sportifs }\end{array}$ & $\begin{array}{l}\text { - Enfants } \\
\text { - Élèves } \\
\text { - Étudiants } \\
\text { - Écoles primaires }\end{array}$ \\
\hline Anglais & $\begin{array}{l}\text { - Positive perception } \\
\text { - Positive attitude } \\
\text { - Positive opinion } \\
\text { - Enjoyment } \\
\text { - Point of view } \\
\text { - Experience }\end{array}$ & $\begin{array}{l}\text { - Sport } \\
\text { - Exercice } \\
\text { - Physical education } \\
\text { - Sport program }\end{array}$ & $\begin{array}{l}\text { - Student } \\
\text { - Children } \\
\text { - Elementary school } \\
\text { - Adolescent }\end{array}$ \\
\hline
\end{tabular}

Étape 4 :

Recherche avancée dans SPORTDiscus

Recherche $1: 441$ articles

Champ 1 : positive perception

Champ 2 : physical activity

Champ 3: student or children

Recherche $3: \mathbf{4 3 5}$ articles

Champ 1 : enjoyment

Champ 2 : physical activity or exercise or sport

Champ 3 : student or children or adolescent
Recherche $2: 4250$ articles

Champ 1: perceptions or attitudes or opinion

Champ 2 : physical activity or exercise or sport Champ 3 : student or children or adolescent

\section{Recherche $4: 446$ articles}

Champ 1: positive perception

Champ 2 : physical activity or exercise or sport

Champ 3: student or children or adolescent

\begin{tabular}{|c|}
\hline $\begin{array}{c}\text { Étape } 5: \\
\text { Sélection des ressources documentaires pertinentes }\end{array}$ \\
\hline $\begin{array}{l}\text { Critères d'inclusion : } \\
\text { - Articles exposant les perceptions d'élèves de } 5 \text { à } 17 \text { ans } \\
\text { - Articles abordant les thèmes en lien avec l'activité physique ou sportive et l'éducation physique } \\
\text { Critères d'exclusion : } \\
\text { - Articles faisant référence au domaine médical } \\
\text { - Articles exposant les perceptions d'acteurs autres que les élèves } \\
\text { - Articles indisponibles en français ou en anglais }\end{array}$ \\
\hline Total : 15 articles \\
\hline$\nabla$ \\
\hline $\begin{array}{c}\text { Étape } 6: \\
\text { Recherche secondaire à partir des ressources documentaires pertinentes }\end{array}$ \\
\hline Total : 4 articles \\
\hline$\nabla$ \\
\hline Nombre final d'articles : 19 \\
\hline
\end{tabular}

Figure 2. Étapes de la recherche documentaire 


\section{eJRIEPS Hors Série 4 Juillet 2021}

\subsection{Analyse documentaire}

Les 19 articles retenus à l'étape de la recherche documentaire ont été analysés selon les étapes proposées par Thomas et Harden (2008). Après avoir été lus, des tableaux résumés ont été créés pour chaque article. Ces tableaux résumés ont permis d'identifier tous les éléments pouvant favoriser une PP de l'AP chez les élèves présents dans les articles. Par la suite, une mise en commun de tous les éléments favorisant une PP de l'AP a été faite. Cela a permis d'identifier 24 facteurs favorisant une PP de l'AP chez les élèves. Enfin, les facteurs dont le sens se ressemble (L'Écuyer, 1990) ou qui sont du même domaine ont été regroupés dans 9 catégories.

\subsection{Stratégie de modélisation avec le modèle de McLeroy et al. (1988)}

Les neuf catégories de facteurs ont été classées dans les différents niveaux du modèle de McLeroy et al. (1988) présenté précédemment. Pour confirmer le classement des catégories de facteurs identifiés dans la littérature dans les niveaux appropriés du modèle socioécologique de McLeroy et al. (1988), un processus de vérification a été réalisé. Un premier classement des catégories a été effectué par le premier auteur et présenté à des experts, soit deux chercheurs dans le domaine de l'activité physique en contexte scolaire. Des discussions ont permis de préciser la définition des catégories de facteurs et d'ajuster le classement de celles-ci dans le modèle de McLeroy et al. (1988). Un consensus a ensuite été atteint concernant la nomenclature et le classement des neuf catégories.

\section{Résultats}

Tout d'abord, le cadre d'analyse modélisé à partir du modèle socio-écologique de McLeroy et al. (1988) est présenté à la figure 3. Toutefois, parmi les facteurs identifiés dans la revue de la littérature, aucun ne se rapporte aux deux derniers niveaux du modèle, soit le niveau des facteurs en lien avec communauté et le niveau des politiques publiques. II semble que les élèves aient davantage soulevé des facteurs qui sont proximaux à eux dans le modèle. Ensuite, les facteurs favorisant une PP de l'AP identifiés à l'étape de l'analyse documentaire sont détaillés et présentés dans l'ordre des trois premiers niveaux du modèle de McLeroy et al. (1988). 


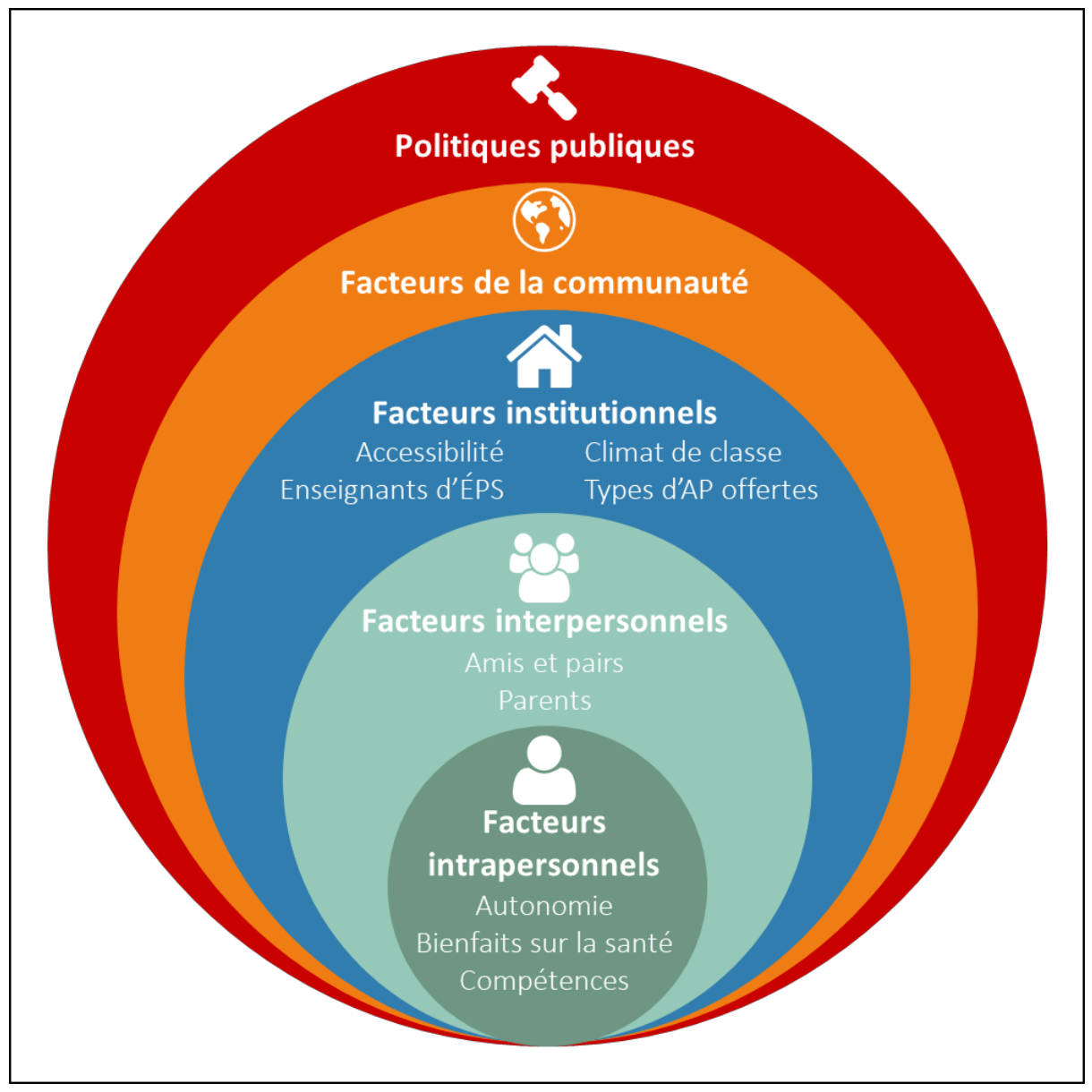

Figure 3. Cadre d'analyse représentant les facteurs favorisant une PP de l'AP chez les élèves

\subsection{Facteurs intrapersonnels}

L'analyse documentaire a permis de relever trois catégories de facteurs intrapersonnels favorisant une PP de l'AP, soit : (1) l'autonomie ; (2) les bienfaits sur la santé ; et (3) les compétences.

\subsubsection{Autonomie}

Sur les 19 études retenues, sept mettent en lumière l'autonomie dans la pratique d'AP. Ce facteur se traduit par : (1) la possibilité de faire des choix et (2) la pratique d'AP à l'extérieur du cadre scolaire.

La possibilité d'avoir une certaine autonomie dans le choix des AP pratiquées est associée à une expérience positive (Hayes, 2017 ; Parker, MacPhail, O’Sullivan, Ni Chróinín, \& McEvoy, 2018). D'ailleurs, dans plusieurs études, un certain nombre d'élèves signalent qu'ils veulent 


\section{eJRIEPS Hors Série 4 Juillet 2021}

faire des AP par choix et en fonction de leurs préférences et non parce qu'ils se les font imposer (Hassandra, Goudas \& Chroni, 2003 ; Van den Berg et al., 2018). Des élèves interrogés dans l'étude d'Harvey et al. (2017) aimeraient avoir la possibilité de donner leur opinion concernant les AP qui sont proposées à l'école ou durant les cours d'EPS. L'étude de Van den Berg et al. (2018) va dans le même sens et ajoute que les élèves désirent que les intervenants prennent en considération leurs idées concernant l'AP à l'école. II semble qu'en pouvant choisir euxmêmes les AP pratiquées et qu'en ayant la chance de faire entendre leurs idées et préférences, les élèves auraient plus de plaisir et seraient plus heureux dans leur pratique d'AP (Coulter \& Woods, 2011 ; James et al., 2018).

L'autonomie dans la pratique d'AP se caractérise aussi par la possibilité de faire des AP à l'extérieur du cadre scolaire. En fait, les élèves considèrent positivement la pratique d'AP à l'extérieur de l'école puisqu'ils peuvent pratiquer l'activité de leur choix au moment et à l'endroit qui leur convient le plus (Parker et al., 2018). D'ailleurs, les résultats de l'étude d'Hassandra et al. (2003) montrent que les élèves qui font des AP à l'extérieur du cadre scolaire sont plus motivés lorsque vient le temps d'en faire à l'école.

\subsubsection{Bienfaits sur la santé}

Six études font valoir l'importance des bienfaits de l'AP sur l'engagement des élèves. Les élèves perçoivent d'ailleurs des bénéfices au niveau des trois dimensions suivantes de leur santé : (1) cognitive ; (2) psychologique ; et (3) physique.

Les bénéfices au niveau de la dimension cognitive de la santé se font ressentir par les élèves lorsqu'ils sont en classe (Van den Berg et al., 2018). Les élèves perçoivent que la pratique d'AP leur permet d'être plus disponibles pour apprendre, c'est-à-dire qu'ils ont une concentration plus soutenue et les idées plus claires.

Les élèves reconnaissent aussi que l'AP est bénéfique pour le maintien d'une bonne santé psychologique. D'abord, il semble que les élèves perçoivent l'AP comme un moyen efficace pour régler les tensions émotionnelles qu'ils ressentent (Everley \& Macfadyen, 2017) et comme une solution pour diminuer leur niveau de stress (Gavin, Mcbrearty, Malo, Abravanel \& Moudrakovski, 2016 ; Van den Berg et al., 2018). Les résultats de l'étude de Bauer et al. (2004) montrent d'ailleurs que l'effet relaxant associé à la pratique d'AP fait partie des raisons pour lesquelles les élèves l'apprécient. De plus, les élèves consultés dans l'étude d'Harvey et al. 


\section{eJRIEPS Hors Série 4 Juillet 2021}

(2017) constatent que la pratique d'AP améliore leur estime et leur confiance personnelles, deux caractéristiques importantes de la santé psychologique.

Enfin, les élèves ressentent que la pratique d'AP est bonne pour leur santé physique et ils se sentent fiers lorsqu'ils accomplissent une activité qui est bénéfique pour leur corps (Harvey et al., 2017). Les élèves questionnés dans l'étude de Van Den Berg et al. (2018) ajoutent que l'AP permet d'avoir une bonne condition physique, de maintenir un poids corporel sain et de renforcir les muscles. D'ailleurs, il semble que la possibilité d'améliorer sa forme physique et son image corporelle soit un aspect motivant à la pratique d'AP chez les filles (Allender, Cowburn \& Foster, 2006).

\subsubsection{Compétences}

Quatorze études sur 19 présentent des résultats en lien avec les compétences des élèves en AP. La catégorie des compétences comprend deux facteurs : (1) la perception favorable de ses compétences et (2) l'amélioration de ses compétences.

En plus d'influencer positivement leur attrait pour l'AP (Eberline et al., 2018 ; Hayes, 2017), la perception d'un bon niveau de compétence favoriserait l'implication et l'engagement des élèves dans plus d'AP et ce, à plus long terme (Casey, Eime, Payne \& Harvey, 2009 ; Garn \& Cothran, 2006; Martinek, Holland \& Seo, 2019 ; Parker et al., 2018). Plusieurs élèves mentionnent qu'ils vont être plus motivés à refaire une AP s'ils se sentent compétents et s'ils sont bons (Everley \& Macfadyen, 2017 ; Harvey et al., 2017). Ces répercussions positives sur le niveau d'AP des élèves seraient dues au fait que les élèves qui ont une perception favorable de leurs compétences sont plus confiants d'atteindre les objectifs fixés par l'enseignant en EPS (Martinek et al., 2019). Garn et Cothran (2006) suggèrent aux enseignants en EPS de proposer des tâches qui valorisent l'amélioration ainsi que l'effort et qui offrent une difficulté optimale pour chacun des élèves. Lorsque les objectifs de la tâche sont orientés vers la maîtrise plutôt que vers la performance, cela augmente le niveau de motivation des élèves à s'y engager, améliore le sentiment de compétence et favorise une expérience positive de l'AP et le plaisir perçu (Coulter \& Woods, 2011 ; Garn \& Cothran, 2006 ; Hassandra et al., 2003; Hayes, 2017 ; Martinek et al., 2019). Les élèves qui ont un bon sentiment de compétence se sentent fiers lorsqu'ils atteignent ces objectifs; toutefois, lorsqu'ils ne réussissent pas à les atteindre, ils comprennent qu'ils doivent continuer à travailler pour s'améliorer (Harvey \& al., 2017). Pour ces élèves, les échecs sont seulement temporaires et ils savent que leurs efforts vont mener au succès. Ils vont donc mettre l'accent sur la résolution de problèmes plutôt que sur leurs 


\section{eJRIEPS Hors Série 4 Juillet 2021}

difficultés. La perception favorable de ses compétences améliore l'estime personnelle de l'élève (Everley \& Macfadyen, 2017).

Les élèves se sentent plus confiants quant à leurs compétences sportives et ressentent plus de plaisir lorsque l'accent est mis sur l'amélioration et non sur la performance (Ni Chróinín \& McMullen, 2019 ; Hassandra et al., 2003). D'ailleurs, les élèves ressentent un sentiment d'accomplissement lorsque les AP favorisent le développement et l'acquisition de nouvelles habiletés motrices (Allender et al., 2006 ; Casey et al., 2009 ; Harvey et al., 2017; Pearson, Irwin \& Burke, 2012). Les élèves interrogés dans l'étude d'Harvey et al. (2017) ajoutent qu'ils se sentent très heureux lorsqu'ils réussissent à améliorer leurs compétences dans une AP ou dans un sport.

\subsection{Facteurs interpersonnels}

L'analyse documentaire a permis de relever deux catégories de facteurs interpersonnels favorisant une PP de l'AP, soit : (1) les amis et les pairs ainsi que (2) les parents.

\subsubsection{Amis et pairs}

Quatorze études sur 19 mettent en évidence l'importance des amis et des pairs dans le développement d'une PP de l'AP chez les élèves du primaire. Cette catégorie comprend quatre facteurs: (1) La participation des amis; (2) la possibilité de s'en faire de nouveaux; (3) la reconnaissance ; et (4) le soutien des amis et des pairs.

II semble qu'il soit très important pour les élèves de faire du sport avec des amis et que cela augmente la motivation et les réussites durant la réalisation d'une AP (Allender et al., 2006 ; Casey et al., 2009 ; Garn \& Cothran, 2006 ; Harvey et al., 2017 ; James et al., 2018 ; Pearson et al., 2012 ; Wanless, Judge, Dieringer \& Johnson, 2017). Le fait de pouvoir socialiser et de passer du temps avec ses amis favorise la participation et l'engagement des jeunes dans l'AP (Bauer et al., 2004 ; Casey et al., 2009 ; Hassandra et al., 2003; Hayes, 2017). D'ailleurs, le fait d'être avec ses amis durant la pratique d'AP augmente le sentiment de confort et de sécurité durant la pratique d'AP puisqu'ils peuvent partager leurs sentiments par rapport aux situations qu'ils vivent (Pearson et al., 2012). Enfin, Everley et Macfayden (2017) stipulent que l'environnement social est le facteur le plus important pour vivre une expérience positive en AP. 


\section{eJRIEPS Hors Série 4 Juillet 2021}

Les situations d'AP sont aussi favorables à la création de nouvelles amitiés, ce qui représente un aspect positif à la pratique d'AP (Allender et al., 2006 ; Carlin et al., 2015 ; Casey et al., 2009; Garn \& Cothran, 2006 ; Gavin et al., 2016).

La reconnaissance et l'approbation des pairs sont une importante source de motivation pour les élèves (Ni Chróinín \& McMullen, 2019 ; Pearson et al., 2012). La reconnaissance des réussites sportives par les pairs favorise les émotions positives comme la satisfaction et la fierté (Ni Chróinín \& McMullen, 2019) en plus d'influencer positivement les élèves qui sont moins actifs et qui hésitent à prendre part aux AP (Ni Chróinín \& McMullen, 2019 ; Van den Berg et al., 2018). Cela encourage les élèves à surmonter les obstacles pour atteindre des objectifs plus ambitieux et à persévérer dans leur pratique d'AP (Casey et al., 2009 ; Ni Chróinín \& McMullen, 2019). Pour les élèves de l'étude d'Everley et Macfadyen (2017), la reconnaissance des pairs signifie aussi faire bonne figure, c'est-à-dire qu'ils aiment faire des AP qui sont hautement évaluées par les autres. Cela leur donne un certain prestige.

Le soutien social est essentiel au développement d'une PP de l'AP (Ni Chróinín \& McMullen, 2019). II se traduit par exemple par le soutien des amis dans l'apprentissage d'un nouveau sport (Everley \& Macfadyen, 2017) ou par l'encouragement de ces derniers à persévérer dans la pratique d'AP (Pearson et al., 2012). Selon Ni Chróinín et McMullen (2019), les amis et les pairs sont tous des sources importantes de soutien social dans la pratique d'AP de l'élève.

\subsubsection{Parents}

Quatre études mettent en lumière l'influence des parents dans le développement d'une PP de l'AP chez les élèves. Cette catégorie comprend en deux facteurs, soit : (1) le soutien des parents dans la pratique d'AP et (2) le comportement des parents.

Le soutien des parents est essentiel dans la pratique d'AP des élèves (Allender et al., 2006 ; Casey et al., 2009). Les parents qui soutiennent leurs enfants dans leur pratique d'AP et qui leur offrent l'opportunité d'en expérimenter une grande diversité contribuent au développement d'une perception favorable de l'AP (Hayes, 2017).

Le comportement des parents semble influencer la perception et les habitudes de leurs enfants concernant la pratique d'AP (Hayes, 2017). Les études de Casey et al. (2009) et de Coulter et Woods (2011) indiquent qu'en plus d'agir comme de bons modèles pour leurs enfants, les parents qui pratiquent des AP de façon régulière contribuent favorablement à l'augmentation du niveau d'AP de leur enfant. 


\section{eJRIEPS Hors Série 4 Juillet 2021}

\subsection{Facteurs institutionnels}

L'analyse documentaire a permis de de relever quatre catégories de facteurs institutionnels, soit : (1) l'accessibilité ; (2) les enseignants en EPS ; (3) le climat de classe ; et (4) les types d'AP offertes.

\subsubsection{Accessibilité}

Des 19 études retenues dans la recherche documentaire, sept présentent des résultats en lien avec la catégorie d'accessibilité. Cette catégorie se divise en trois facteurs : (1) l'accès à des équipements et des installations de qualité; (2) les faibles coûts d'accès ; et (3) l'inclusivité des équipes sportives à l'école.

L'accessibilité à des équipements et à des installations sportives de qualité favorise le développement d'une PP quant à l'AP puisqu'elle favorise la participation et la motivation des élèves à prendre part à des AP (Hassandra et al., 2003). L'accessibilité se traduit par la disponibilité, la quantité et la proximité des équipements ainsi que des installations sportives (Carlin et al., 2015; Casey et al., 2009; Harvey et al., 2017 ; Hassandra et al., 2003 ; James et al., 2018). Par exemple, les élèves interrogés dans l'étude de James et al. (2018) mentionnent qu'ils sont plus enclins à utiliser les installations sportives lorsqu'elles sont localement disponibles, c'est-à-dire lorsqu'elles sont facilement accessibles à partir de la maison, puisqu'ils peuvent les utiliser lorsqu'ils le désirent sans attendre le transport de leurs parents.

Les AP qui sont gratuites ou peu coûteuses et qui nécessitent un équipement abordable sont plus attrayantes pour les élèves (Carlin et al., 2015 ; James et al., 2018). Lorsqu'ils ont la possibilité d'expérimenter une nouvelle AP, ils ne veulent pas avoir à débourser beaucoup d'argent puisqu'ils ne savent pas s'ils vont réellement l'apprécier. Autrement, ils considéreraient cela comme une perte d'argent (Carlin et al., 2015). D'ailleurs, les élèves ne veulent pas dépenser beaucoup d'argent pour être physiquement actifs afin d'avoir les moyens de participer plus souvent aux AP offertes par l'école ou les centres sportifs (James et al., 2018). Les élèves semblent s'entendre pour dire qu'ils ont un désir plus fort de s'engager dans des AP qui sont abordables. Le faible coût d'accès aux AP apparaît comme un facteur favorable au développement d'une PP de l'AP chez les élèves (James et al., 2018).

La facilité d'accès aux équipes sportives favorise aussi le développement d'une PP de l'AP. Les résultats de l'étude de Bauer et al. (2004) montrent qu'il est parfois difficile pour les élèves de pouvoir faire partie d'une équipe sportive, soit parce que les tests physiques pour y accéder 


\section{eJRIEPS Hors Série 4 Juillet 2021}

sont trop difficiles ou parce que les élèves n'ont pas le talent et les habiletés requises. Toutefois, lorsque les critères pour intégrer une équipe sportive à l'école sont basés sur l'effort et la volonté plutôt que sur le talent, les élèves se sentent encouragés et valorisés (Bauer et al., 2004). Ces critères, plus inclusifs, facilitent l'accès aux équipes sportives (Ni Chróinín \& McMullen, 2019).

\subsubsection{Enseignants en éducation physique et à la santé}

L'enseignant en EPS joue un rôle crucial dans le développement de la PP de l'AP chez les élèves. Sur les 19 études recensées, neuf identifient que : (1) l'attitude et le comportement de l'enseignant; (2) les rétroactions qu'il donne ; et (3) le style d'enseignement auquel il adhère influencent la perception des élèves envers l'AP.

L'attitude et le comportement de l'enseignant en EPS influencent significativement la motivation des élèves dans la pratique d'AP (Hassandra et al., 2003 ; Wanless et al., 2017). Casey et al. (2009) décrivent l'impact que l'enseignant en EPS peut avoir chez ses élèves. En fait, lorsque l'enseignant agit comme un bon modèle qui promeut un mode de vie sain et actif, il encourage ses élèves à être plus actifs. Lorsque ce dernier est amical, amusant, souriant, dynamique, équitable, empathique, disponible et qu'il participe aux AP qu'il propose, les élèves ont un intérêt plus grand pour les cours d'EPS et ont plus de plaisir dans la pratique d'AP (Carlin \& al., 2015 ; Garn \& Cothran, 2006 ; Hassandra et al., 2003). L'enseignant doit aussi montrer qu'il croit en chaque élève pour leur donner confiance (Pearson et al., 2012). L'étude de Coulter et Woods (2011) va dans le même sens et ajoute que la présence d'un enseignant en EPS bienveillant améliore de façon considérable les croyances de l'enfant quant à ses compétences sportives et à son estime personnelle. De plus, les élèves soulignent l'importance d'avoir un enseignant qui est passionné par son travail puisque cela a un effet sur sa façon d'être avec eux (Garn \& Cothran, 2006).

Les enseignants en EPS qui fournissent des rétroactions positives aux élèves les encouragent à travailler plus fort et à persévérer dans leur pratique d'AP (Casey et al., 2009). L'étude d'Hayes (2017) va le même sens et ajoute que les renforcements émis de façon régulière améliorent l'expérience des élèves lorsqu'ils font des AP.

Selon Hayes (2017), les styles d'enseignement individualisés où les élèves ont la possibilité de faire des choix et où il y a possibilité de mettre en place des objectifs personnels sont favorables au développement d'une PP de l'AP. L'enseignant doit s'assurer que tous les élèves puissent 


\section{eJRIEPS Hors Série 4 Juillet 2021}

s'impliquer activement dans les tâches qu'il propose, et ce, sans qu'ils se sentent embarrassés (Allender et al., 2006 ; Hayes, 2017). De plus, les élèves apprécient particulièrement lorsque l'enseignant prend le temps de bien expliquer et de bien démontrer les tâches qui doivent être réalisées (Hassandra et al., 2003).

\subsubsection{Climat de classe}

Quatre études mettent de l'avant des résultats en lien avec le climat de classe. Trois facteurs sont mis en évidence, soit : (1) les classes non mixtes; (2) l'absence de compétition ; et (3) la sécurité.

Pour différentes raisons, certains élèves désirent faire partie de classes d'EPS non mixtes, c'est-à-dire qu'ils aimeraient que les filles et les garçons soient séparés. Ce désir se manifeste surtout chez les filles qui mentionnent qu'elles seraient plus à l'aise de participer durant les cours d'EPS et qu'elles se sentiraient moins exclues en étant séparées des garçons (Casey et al., 2009). En plus de se sentir gênée durant les cours d'EPS (Bauer et al., 2004), il semble que les filles se font souvent taquiner par ces derniers sur leur image corporelle, sur leurs compétences sportives et sur leur performance (Casey et al., 2009).

L'absence de compétition est un facteur important pour développer une PP de l'AP. Bien que la présence d'une compétition saine durant les cours d'EPS puisse favoriser l'engagement de certains élèves, la plupart d'entre eux s'entendent pour dire que la pratique d'AP est beaucoup plus agréable lorsqu'il y a absence de compétition et lorsque l'objectif de l'activité n'est pas de gagner, mais plutôt de s'améliorer et de maîtriser la tâche (Allender et al., 2006). De cette façon, les élèves ressentent moins de pression pour performer et ils ont plus de plaisir (Allender et al., 2006 ; Casey et al., 2009).

La pratique d'AP dans un environnement sécuritaire apparaît aussi comme un aspect favorable pour pratiquer des AP chez les élèves (Allender et al., 2006). Les élèves de l'étude de Van den Berg et al. (2018) mentionnent qu'ils se sentent plus à l'aise dans leur pratique d'AP lorsqu'il y a présence d'un adulte qui assure leur sécurité.

\subsubsection{Types d'activités physiques offertes}

Finalement, huit études présentent des résultats en lien avec les types d'AP offertes aux élèves. Cette catégorie se divise en deux facteurs : (1) les AP en lien avec les intérêts des élèves et (2) la variété des AP offertes. 


\section{eJRIEPS Hors Série 4 Juillet 2021}

Dans un premier temps, les élèves qui ont la possibilité de pratiquer des AP en lien avec leurs intérêts ont une plus grande motivation à y prendre part (Hassandra et al., 2003). Par exemple, les AP qui font intervenir la dimension cognitive, qui permettent de vivre des expériences physiques ou sensorielles particulières (Everley \& Macfadyen, 2017) ou qui incluent les nouvelles technologies (Carlin et al., 2015) emballent un certain nombre d'élèves. Dans l'étude de Carlin et al. (2015), les élèves mentionnent que les AP qui respectent les intérêts plus spécifiques aux filles et aux garçons sont plus amusantes. Par exemple, certaines filles dans l'étude de James et al. (2018) aimeraient avoir la possibilité de faire des AP plus artistiques comme le cheerleading.

Enfin, les élèves mentionnent que, plus les AP offertes à l'école sont variées et originales, plus elles sont amusantes et motivantes à long terme (Allender et al., 2006 ; Carlin et al., 2015 ; James et al., 2018 ; Parker et al., 2018 ; Van den Berg et al., 2018). II semble aussi que les élèves se considérant moins sportifs soient intéressés par des AP plus accessibles telles que la marche ou la danse (Carlin et al., 2015). Les élèves interrogés dans l'étude d'Harvey et al. (2017) mentionnent qu'ils aiment particulièrement apprendre de nouveaux jeux. II semble aussi que la découverte de nouvelles AP incite les élèves à s'engager dans les cours d'EPS (Hassandra et al., 2003).

\section{Discussion}

Le présent article visait à (1) identifier les facteurs qui favorisent une PP de l'AP chez les élèves du primaire et à (2) modéliser un cadre d'analyse permettant de catégoriser ces facteurs afin de mieux comprendre la façon dont ils se déploient autour de l'élève. La discussion qui suit a pour but de mettre en lumière et d'interpréter certains facteurs qui apparaissent avec une occurrence plus forte dans la littérature et d'énoncer certaines recommandations pour favoriser le développement d'une PP de I'AP en milieu scolaire chez les élèves du primaire.

\subsection{Occurrence des facteurs}

Bien que les facteurs identifiés dans la recherche documentaire semblent tous avoir une influence considérable sur les perceptions des élèves quant à l'AP, certains facteurs apparaissent plus fréquemment dans la littérature : les amis, les enseignants en EPS, les compétences, les types d'AP offertes et l'autonomie. L'occurrence de ces facteurs permet de dégager trois constats, soit que : (1) les individus qui interagissent directement avec les élèves, 


\section{eJRIEPS Hors Série 4 Juillet 2021}

(2) le sentiment d'efficacité personnelle ainsi que (3) les intérêts des élèves sont des éléments de grande importance dont il faut se préoccuper pour que les élèves puissent développer une perception favorable de l'AP.

\subsubsection{Individus qui interagissent directement avec les élèves}

Les individus qui interagissent directement avec les élèves en milieu scolaire lorsqu'ils sont en situation d'AP, à savoir les amis et les pairs ainsi que les enseignants en EPS, semblent exercer une influence importante sur leur perception de l'AP. En effet, ils auraient la capacité de la moduler (Hayes, 2017). Selon cet auteur, ces individus ont la capacité de favoriser le développement d'une PP de l'AP seulement si une ambiance de bienveillance et de soutien se fait ressentir. Le soutien social et les relations que créent les élèves avec des adultes signifiants, comme l'enseignant en EPS, et avec leurs amis et leurs pairs, influencent grandement leur motivation à s'engager dans la pratique d'une AP (Stuntz \& Weiss, 2010). D'ailleurs, il semble que les amis et les pairs influencent la pratique d'AP selon six facteurs, soit : (1) le soutien reçu ; (2) la présence des amis ; (3) les croyances et les normes établies par les pairs ; (4) la qualité des relations avec les pairs ; (5) le sentiment d'appartenance à un groupe ; et (6) l'intimidation vécue (Fitzgerald, Fitzgerald \& Aherne, 2012). Ces éléments nous permettent de mieux comprendre pourquoi les élèves mettent en lumière l'importance d'être entourés d'individus avec qui ils entretiennent une bonne relation et avec qui ils se sentent à l'aise. Parmi les facteurs soulevés par les élèves on retrouve notamment l'importance d'être entouré de ses amis et de ses pairs dans la pratique d'AP (Harvey et al., 2017 ; James et al., 2018) et l'importance d'être en présence d'un enseignant en EPS amical, empathique et bienveillant (Carlin et al., 2015 ; Garn \& Cothran, 2006 ; Hassandra et al., 2003). Ces exemples supportent tout à fait les propos d'Allen (2003) qui stipule que les interactions sociales que vit l'élève à travers sa pratique d'AP influencent la qualité de son expérience. Cette influence serait d'ailleurs encore plus grande auprès des élèves du primaire qui se rapprochent de l'adolescence (Hayes, 2017).

\subsubsection{Sentiment d'efficacité personnelle}

Tout comme Harvey et al. (2017) et Stuntz et Weiss (2010), qu'en plus d'être un facteur important à l'engagement des élèves dans la pratique d'AP, le sentiment d'efficacité personnelle contribue au développement d'une PP de l'AP chez ces derniers. Le sentiment 


\section{eJRIEPS Hors Série 4 Juillet 2021}

d'efficacité personnelle se définit comme étant l'évaluation qu'une personne fait de sa capacité à exécuter les actions pour accomplir une tâche (Bandura, 1986). Cette théorie explique pourquoi les élèves mentionnent, dans plusieurs études, l'importance de se sentir compétent dans leur pratique d'AP en plus d'être valorisé par leurs pairs (Weiss, 2000). À titre d'exemples, les élèves se disent plus heureux et plus motivés lorsqu'ils parviennent à améliorer leurs compétences sportives (Harvey et al., 2017), ils accordent beaucoup de valeur à la reconnaissance de leurs réussites sportives par leurs pairs (Ni Chróinín \& McMullen, 2019) et ils apprécient particulièrement les rétroactions positives et fréquentes de la part de leur enseignant en EPS (Casey et al., 2009). Les recherches montrent d'ailleurs que la façon dont l'enseignant en EPS donne ses rétroactions peut influencer la façon dont l'élève va interpréter sa performance et son niveau de motivation pour ses prochaines expériences d'AP (Biddle, Hanrahan, \& Sellars, 2001). En référence aux rétroactions, Bandura (1994) mentionne qu'à travers les suggestions, les conseils et les questionnements de la personne ressource, soit l'enseignant en EPS, les élèves pourraient être amenés à croire qu'ils possèdent le potentiel pour accomplir une tâche. L'influence de ces rétroactions sur le sentiment d'efficacité personnelle dépend toutefois de l'expertise et de la crédibilité de la personne ressource (Rondier, 2003). Ce besoin d'être valorisé et de compétence est à considérer dans les interventions qui sont mises en place dans les écoles primaires. À cet effet, il apparait important que les élèves puissent expérimenter des AP qui leur permettent de vivre des succès afin de développer une PP de l'AP et d'être guidé et encouragé de façon constructive par leurs pairs, leur enseignant d'EPS ainsi que par les autres intervenants qui les entourent.

\section{1 .3 ; Intérêts des élèves}

Les intérêts des élèves doivent être priorisés pour leur faire vivre une expérience d'AP positive (Hayes, 2017 ; Parker et al., 2018). Cette nécessité de considérer les intérêts des élèves se retrouve dans plusieurs facteurs identifiés dans la recherche documentaire. Dans les études de Carlin et al. (2015) et de James et al. (2018), les élèves sont plus motivés et le plaisir est accentué lorsque les AP sont en lien avec leurs intérêts. La nécessité de considérer les intérêts des élèves présente un lien avec le besoin d'autonomie dans leur pratique d'AP. Ce besoin se traduit notamment par le désir de choisir les AP qu'ils pratiquent en fonction de leurs préférences (Hassandra et al., 2003 ; Van den Berg et al., 2018) et par l'intérêt que les élèves portent à la pratique d'AP à l'extérieur du cadre scolaire à cause de l'autonomie dont ils 


\section{eJRIEPS Hors Série 4 Juillet 2021}

bénéficient pour décider des modalités des AP qu'ils pratiquent (Parker et al., 2018). Ces exemples rejoignent les conclusions de Coulter et Woods (2011), d'Harvey et al. (2017), de James et al. (2018) et de Van den Berg et al. (2018) selon lesquelles il est essentiel de donner une voix aux élèves, c'est-à-dire de leur laisser la possibilité de faire des choix lorsque vient le temps de faire des AP à l'école et de les impliquer dans la mise en œuvre des interventions en lien avec l'AP à l'école.

\subsection{Recommandations pour favoriser le développement d'une perception positive de l'activité physique chez les élèves}

L'identification des facteurs favorisant une PP de l'AP chez les élèves du primaire ainsi que la modélisation du cadre d'analyse montrent que les stratégies d'intervention visant à rendre les élèves actifs en milieu scolaire doivent être adaptées à différents niveaux pour assurer le développement d'une PP de l'AP. À cet effet, des recommandations sont proposées aux enseignants en EPS, aux dirigeants scolaires ainsi qu'aux chercheurs qui se retrouvent dans des positions privilégiées pour élaborer et améliorer les stratégies d'interventions en lien avec l'AP en milieu scolaire.

\subsubsection{Enseignants en éducation physique et à la santé}

L'enseignant en EPS joue un rôle crucial auprès de l'élève. II est d'ailleurs reconnu comme étant l'acteur du milieu scolaire qui a le plus d'impact dans le développement de la littératie physique des élèves (EPS Canada, 2009 ; Hayes, 2017) ainsi que sur leurs comportements et leurs perceptions en lien avec l'AP (Hayes, 2017 ; Stuntz \& Weiss, 2010). Par son contact avec les élèves, il a le privilège de pouvoir les impliquer directement dans l'élaboration des AP offertes à l'école. Ainsi, l'enseignant en EPS se doit d'assurer la représentativité de l'ensemble des élèves dans le choix des AP qui sont proposées à l'école, soit en questionnant les élèves à propos de ce qu'ils désirent faire comme AP à l'école et en démontrant aux élèves que leurs idées sont les bienvenues. Les élèves questionnés dans les études mentionnent aussi qu'ils sont plus motivés à faire des AP lorsqu'ils se sentent bons et compétents (Everley \& Macfadyen, 2017 ; Harvey et al., 2017). À cet effet, l'enseignant d'EPS doit s'assurer que tous les élèves puissent vivre des réussites dans leur pratique d'AP (Casey et al., 2009). Il est donc de sa responsabilité de développer des situations d'AP qui valorisent l'amélioration, l'effort et l'apprentissage afin que les élèves puissent développer un bon sentiment d'efficacité 


\section{eJRIEPS Hors Série 4 Juillet 2021}

personnelle. Ainsi, l'enseignant en EPS doit encourager ses élèves à persévérer lorsqu'ils rencontrent des difficultés tout en respectant les limites de chacun. Puisque les interactions sociales jouent un grand rôle dans le développement de la perception de l'AP (Smith, 1999), il est essentiel que l'enseignant désapprouve et élimine toute forme de marque de non-respect, d'intimidation ou de compétition malsaine entre les élèves. L'enseignant doit s'assurer que le climat qui règne lors de la pratique d'AP à l'école soit positif. Enfin, il apparait important que l'enseignant en EPS s'implique dans l'école pour transmettre sa passion pour l'AP et pour mettre en place des projets qui stimuleront et donneront envie aux élèves de s'engager dans la pratique d'AP. Par son implication, l'enseignant en EPS favorise le développement d'un environnement actif significatif en collaboration avec les autres intervenants scolaires et permet aux élèves de consolider leurs apprentissages en lien avec l'AP.

\subsubsection{Dirigeants scolaires}

Les programmes scolaires rattachés à la pratique d'AP devraient prioriser le développement d'une PP de l'AP chez les élèves, notamment parce qu'ils pourraient avoir des répercussions à plus long terme sur leur mode de vie actif (Burns et al., 2017). Comme les élèves montrent clairement qu'ils veulent une part du pouvoir dans les décisions qui concernent leur pratique d'AP, les dirigeants scolaires devraient les impliquer dans l'élaboration et le remaniement des programmes relatifs à l'AP en milieu scolaire. Les intérêts et les besoins des élèves doivent donc être considérés comme une priorité et mis de l'avant dans les programmes scolaires. Dans un autre ordre d'idée, l'accessibilité est aussi un facteur important relevé par les élèves (Carlin et al., 2015 ; Harvey et al., 2017 ; James et al., 2018). Si l'on souhaite rendre accessible des équipements et des installations sportives de qualité ainsi que des activités organisées abordables aux élèves, il serait nécessaire de prioriser la création d'un environnement actif à l'école, soit en améliorant la cour d'école, en achetant du matériel sportif plus attrayant ou en offrant des AP parascolaires gratuites. Les milieux scolaires devraient aussi prendre des ententes avec les municipalités afin d'avoir accès plus facilement et à moindre coût aux installations sportives de la ville. Ainsi, les élèves pourraient pratiquer des AP abordables et à proximité.

\subsubsection{Chercheurs}




\section{eJRIEPS Hors Série 4 Juillet 2021}

Les perceptions des élèves sont sous-représentées dans la littérature scientifique et peu d'études questionnent directement les élèves à propos des facteurs qui influencent leur pratique d'AP (Carlin et al., 2015). Ainsi, il serait bénéfique de mettre davantage l'élève au centre des préoccupations de la communauté scientifique puisque ces derniers sont la cible des interventions en lien avec l'AP en milieu scolaire. Selon Jacquez et al. (2013), l'implication des élèves dans les recherches peut non seulement améliorer la qualité des recherches, mais aussi augmenter les chances que les résultats soient considérés et mis en pratique dans des contextes réels. Les chercheurs auraient donc avantage à intégrer les élèves à leurs recherches et à investiguer leurs perceptions en utilisant le cadre d'analyse inspiré du modèle socio-écologique de McLeroy et al. (1988) présenté dans cet article. Par exemple, au Québec, de futures recherches pourraient porter sur les perceptions des élèves pour mieux comprendre les retombées et pour améliorer la mise en œuvre de la mesure 15023 - « À l'école, on bouge! ». Enfin, il serait intéressant que la communauté scientifique se penche sur l'évaluation de programmes d'AP en milieu scolaire qui promeuvent le développement d'une PP de l'AP et ainsi observer les répercussions à long terme de ces programmes sur le mode de vie actif des élèves.

\section{Conclusion}

Cet article a permis d'identifier les facteurs favorisant une PP de l'AP chez les élèves du primaire et de modéliser un cadre d'analyse permettant de catégoriser ces facteurs. D'ailleurs, ce dernier apparait pertinent pour identifier, décrire et caractériser les perceptions des élèves dans le contexte où des stratégies d'interventions visant à augmenter leur niveau d'AP sont mises en place en milieu scolaire. De plus en s'inspirant du cadre d'analyse et en mettant de l'avant les facteurs qui caractérisent une PP de l'AP chez les élèves, les intervenants scolaires pourront mieux cibler leurs interventions afin qu'elles puissent avoir un impact plus signifiant sur le mode de vie actif des élèves. Ces interventions qui favorisent une PP de I'AP contribueront à l'engagement des jeunes dans la pratique d'AP et favoriseront l'adoption d'un mode de vie actif à plus long terme chez les élèves. 


\section{eJRIEPS Hors Série 4 Juillet 2021}

\section{Bibliographie}

Allender, S., Cowburn, G., \& Foster, C. (2006). Understanding participation in sport and physical activity among children and adults: A review of qualitative studies. Health Education Research, 21(6), 826-835.

Bandura, A. (1986). Social foundations of thought and action: A social cognitive theory. Englewood Cliffs, New Jersey: Prentice-Hall.

Bandura, A. (1994). Self-efficacity. Encyclopedia of human Behavior, 4, 71-81.

Bauer, K.W., Yang, Y.W., \& Austin, S.B. (2004). "How Can We Stay Healthy when you're Throwing All of this in Front of Us?" Findings from Focus Groups and Interviews in Middle Schools on Environmental Influences on Nutrition and Physical Activity. Health Education \& Behavior, 31(1), 34-46.

Biddle, S., Hanrahan, S., \& Sellars, C. (2001). Attributions: Past, present, and future. Dans R. Singer, H. Hausenblas et C. Janelle (dir.), Handbook of sport psychology ( $2^{\mathrm{e}}$ éd., p. 444-471). John Wiley \& Sons.

Bouchard, C. (1987). Intervenir à partir de l'approche écologique : au centre, l'intervenante. Service social, 36 (2-3), 454-477.

Bronfenbrenner, U. (1977). Toward an experimental ecology of human development. American Psychologist, 32(7), 513.

Brunton, G., Harden, A., Rees, R., Kavanagh, J., Oliver, S., \& Oakley, A. (2003). Children and Physical Activity: A systematic review of barriers and facilitators. Angleterre, Londres : Université de Londres.

Burns, R.D., Fu, Y., \& Podlog, L.W. (2017). School-based physical activity interventions and physical activity enjoyment: A meta-analysis. Preventive Medicine, 103, 84-90.

Cairney, J., Kwan, M.Y.W., Velduizen, S., Hay, J., Bray, S.R., \& Fought, B.E. (2012). Gender, perceived competence and the enjoyment of physical education in children: $A$ longitudinal examination. International Journal of Behavioral Nutrition and Physical Activity, 9(26). https://doi.org/10.1186/1479-5868-9-26

Cardinal, B.J., Yan, Z., \& Cardinal, M. (2013). Negative Experiences in Physical Education and Sport: How Much Do They Affect Physical Activity Participation Later in Life? Journal of Physical Education, Recreation \& Dance, 84(3), 49-53. 


\section{eJRIEPS Hors Série 4 Juillet 2021}

Carlin, A., Murphy, M.H., \& Gallagher, A.M. (2015). Current influences and approaches to promote future physical activity in 11-13 years olds: a focus group study. BMC Public Health, 15(1), 1270.

Carson, V., Hunter, S., Kuzik, N., Gray, E.C, Poitras, V.J., Chaput, J.-P., Saunders, T.J., Katzmarzyk P.T., Okely, A.D., Connor Gorber, S., Kho, M.E., Sampson, M., Lee, H., \& Tremblay, M.S. (2016). Systematic review of sedentary behaviour and health indicators in school-aged children and youth: An update. Applied Physiology, Nutrition, and Metabolism, 41(6), 240-265.

Casey, M.M., Eime, R.M., Payne, W.R., \& Harvey, J.T. (2009). Using a Socioecological Approach to Examine Participation in Sport and Physical Activity Among Rural Adolescent Girls. Qualitative Health Research, 19(7), 881-893.

Chu, C.H., Chen, F.T., Pontifex, M., Sun, Y., \& Chang, Y.K. (2019). Health-related physical fitness, academic achievement, and neuroelectric measures in children and adolescents. International Journal of Sport and Exercise Psychology, 17(2), 117-132.

Coalition québécoise sur la problématique du poids. (2013). 5 recommandations pour permettre aux jeunes d'être plus actifs à l'école. Repéré à http://www.cqpp.qc.ca/documents/file/2013/5-recommandations-jeunes-actifsecole 2013-02.pdf

Comité scientifique de Kino-Québec. (2011). L'activité physique, le sport et les jeunes - Savoir et agir. Repéré à http://www.kino-quebec.qc.ca/

Coulter, M. \& Woods, C.B. (2011). An exploration of children's perceptions and enjoyment of school-based physical activity and physical education. Journal of Physical Activity \& Health, 8(5), 645-654.

Devís-Devís, J., Beltrán-Carrillo, V. J., \& Peiró-Velert, C. (2015). Exploring socio-ecological factors influencing active and inactive Spanish students in years 12 and 13. Sport, Education and Society, 20 (3), 361-380. doi: 10.1080/13573322.2012.754753

Dobbins, M., Husson, H., DeCorby, K., \& LaRocca, R. L. (2013). School-based physical activity programs for promoting physical activity and fitness in children and adolescents aged 6 to 18.

Repéré

https://www.cochranelibrary.com/cdsr/doi/10.1002/14651858.CD007651.pub2/epdf/ful 


\section{eJRIEPS Hors Série 4 Juillet 2021}

Eberline, A., Judge, L.W., Walsh, A., \& Hensley, L.D. (2018). Relationship of Enjoyment, Perceived Competence, and Cardiorespiratory Fitness to Physical Activity Levels of Elementary School Children. The Physical Educator, 75, 394-413.

Eddolls, W.T.B., McNarry, M.A., Lester, L., Winn, C.O.N., Stratton, G., \& Mackintosh, K.A. (2018). The association between physical activity, fitness and body mass index on mental well-being and quality of life in adolescents. Quality of Life Research, 27, 2313-2320.

EPS Canada. (2009). Énoncé de position. Le savoir-faire physique à l'intention des enseiganntes et enseignants. Repéré à https://epscanada.ca/sites/default/files/content/docs/resources/PhysicalLiteracyPositionPaperFI NAL FR.pdf

Everley, S. \& Macfadyen, T. (2017). 'I like playing on my trampoline; it makes me feel alive.' Valuing physical activity: Perceptions and meanings for children and implications for primary schools. Education 3-13, 45(2), 151-175.

Fitzgerald, A., Fitzgerald, N., \& Aherne, C. (2012). Do peers matter? A review of peer and/or friends' influence on physical activity among American adolescents. Journal of Adolescence, 35, 941-958.

Gadais, T. (2015). Les stratégies d'intervention pour aider les jeunes à gérer leur pratique d'activité physique. Une revue de la littérature. Staps, 3(109), 57-77.

Garn, A. C. \& Cothran, D. J. (2006). The fun factor in physical education. Journal of Teaching in Physical Education, 25, 281-297.

Gavin, J., Mcbrearty, M., Malo, K., Abravanel, M., \& Moudrakovski, T. (2016). Adolescents' Perception of the Psychosocial Factors affecting Sustained Engagement in Sports and Physical Activity. International Journal of Exercise Science, 9(4), 384-411.

Gouvernement du Québec (2018). Document d'information complémentaire à la mesure 15023 - À l'école, on bouge au cube ! Québec, QC : Ministère de l'Éducation et de l'Enseignement supérieur. Repéré à http://www.education.gouv.qc.ca/fileadmin/site web/documents/loisir-sport/Infocomp-mesure15023-sept2018.pdf

Grenier, J. (2006). Description de l'habitude de pratique régulière d'activités physiques des étudiants de niveau collégial. Cégep du Vieux Montréal. https://archipel.uqam.ca/319/1/PAREAGRENIER.pdf 


\section{eJRIEPS Hors Série 4 Juillet 2021}

Guddal, M.H., Stensland, S.Ø., Småstuen, M.C., Johnsen, M.B., Zwart, J.A., \& Storheim, K. (2019). Physical activity and sport participation among adolescents: associations with mental health in different age groups. Results from the Young-HUNT study: A crosssectional survey. BMJ Open, 9. https://doi.org/10.1136/bmjopen-2018-028555

Guthold, R., Stevens, G.A., Riley, A.M., \& Bull, F.C. (2020). Global trends in insufficient physical activity among adolescents: a pooled analysis of 298 population-based surveys with 1.6 million participants. The Lancet Child and Adolescent Health, 4, 23-35.

Harvey, J., Pearson, E.S., Sanzo, P., \& Lennon, A. E. (2017). Exploring the perspectives of 10, 11-, and 12-year-old primary school students on physical activity engagement "'Cause you can't just be sitting at a desk all the time!". Child: Care, Health and Development, 44(3), 433-442.

Hassandra, M., Goudas, M., \& Chroni, S. (2003). Examining factors associated with intrinsic motivation in physical education: a qualitative approach. Psychology of Sport and Exercice, 4, 211-223.

Hayes, D. (2017). The love of sport: An investigation into the perceptions and experiences of physical education amongst primary school pupils. Research Papers in Education, 32(4), 518-534.

Hyndman, B. (2016). A Qualitative Investigation of Australian Youth Perceptions to Enhance School Physical Activity: The Environmental Perceptions Investigation of Children's Physical Activity (EPIC-PA) Study. Journal Of Physical Activity \& Health, 13(5), 543550.

Institut de la statistique du Québec (2015). Les activités sédentaires chez les jeunes : qui les pratique et quelle en est l'évolution depuis 2007? Repéré à http://www.stat.gouv.qc.ca/statistiques/sante/bulletins/zoom-sante-201504.pdf

Jacquez, F., Vaughn, L.M., \& Wagner, E. (2013). Youth as partners, participants or passive recipients: A review of children and adolescents in community-based participatory research (CBPR). American Journal of Community Psychology, 51(1), 176-189.

James, M., Todd, C., Scott, S., Stratton, G., McCoubrey, S., Christan., D., ..., Brophy, S. (2018). Teenage recommendations to improve physical activity for their age group: A qualitative study. BMC Public Health, 18(1), 372. https://doi.org/10.1186/s12889-018$\underline{5274-3}$ 


\section{eJRIEPS Hors Série 4 Juillet 2021}

L'Écuyer, R. (1990). Méthodologie de l'analyse développementale de contenu : Méthode GPS et concept de soi. Québec, Québec : Presses de l'Université du Québec.

Legendre, R. (2005). Dictionnaire de l'éducation (3e éd.). Montréal : Guérin.

Martinek, T., Holland, B., \& Seo, G. (2019) Understanding Physical Activity Engagement in Students: Skills, Values, and Hope. Revista Internacional de Ciencias del Deporte, 55, 88-101.

McLeroy, K.R., Bibeau, D., Steckler, A., \& Glanz, K. (1988). An ecological perspective on health promotion programs. Health education quarterly, 15(4), 351-377.

Metcalf, B., Henley, W., \& Wilkin, T. (2012). Effectiveness of intervention on physical activity of children: Systematic review and meta-analysis of controlled trials with objectively measured outcomes. British Medical Journal, 345. https://doi.org/10.1136/bmj.e5888

Moeijes, J., Van Busschbach, J.T., Bosscher, R.J., \& Twisk, J.W.R. (2018). Sports participation and psychosocial health: a longitudinal observational study in children. BMC Public Health, 18, 702. https://doi.org/10.1186/s12889-018-5624-1

Ni Chróinín, D. \& McMullen, J. (2019). 'The world is a happier place': Celebration in a wholeof-school physical activity initiative. European Physical Education Review, 26(2), 1-16.

Organisation mondiale de la Santé (OMS). (2010). Recommandations mondiales sur l'activité physique pour la santé. Repéré à https://apps.who.int/iris/bitstream/handle/10665/44436/9789242599978 fre.pdf;jsessi onid=FF5794F9608E3981CDDAAE85445FAA69? sequence $=1$

Organisation mondiale de la Santé (OMS). (2019). Le rôle de l'école. Repéré à https://www.who.int/dietphysicalactivity/childhood schools/fr/

Parker, M., MacPhail, A., O'Sullivan, M., Ni Chróinín, D., \& McEvoy, E. (2018). 'Drawing' conclusions: Irish primary school children's understanding of physical education and physical activity opportunities outside of school. European Physical Education Review, 24(4), 449-466.

ParticipACTION. (2020). Bulletin de l'activité physique chez les enfants et les jeunes de ParticipACTION. Repéré

https://participaction.cdn.prismic.io/participaction/3b498307-98c1-4210-815569322766799f Bulletin complet.pdf 


\section{eJRIEPS Hors Série 4 Juillet 2021}

Pate, R.R., Davis, M.G., Robinson, T.N., Stone, E.J., McKenzie, T.L., \& Young, J.C. (2006).

Promoting physical activity in children and youth: A leadership role for schools. Circulation, 114(11), 1214-1224.

Pearson, E.S., Irwin, J.D., \& Burke, S.M. (2012). The Children's Health and Activity Modification Program (C.H.A.M.P.): Participants' perspectives of a four-week lifestyle intervention for children with obesity. Journal of Child Health Care, 16(4), 382-394.

Poitras, V.J., Gray, C.G, Borghese, M.M., Carson, V., Chaput, J.-P., Janssen, I, Katzmarzyk, P.T., Pate, R.R., Connor Gorber, S., Kho, M.E., Sampson, M., \& Tremblay, M. (2016). Systematic review of the relationships between objectively measured physical activity and health indicators in school-aged children and youth. Applied Physiology, Nutrition, and Metabolism, 41(6), 197-239.

Pontifex, M.B., Parks, A.C., Henning, D.A., \& Kamijo, K. (2015). Single Bouts of Exercise Selectively Sustain Attentional Processes. Psychophysiology, 52(5), 618-625.

Richard, L., Barthélémy, L., Tremblay, M.C., Pin, S., \& Gauvin, L. (2013). Interventions de prévention et promotion de la santé pour les aînés : modèle écologique. Guide d'aide à l'action francoquébécois. Saint-Denis : Inpes.

Rondier, M. (2003). A. Bandura. Auto-efficacité. Le sentiment d'efficacité personnelle. Open Edition Journals, 33(3), 475-476.

Sallis, J.F., Owen, N., \& Fisher, E.B. (2008). Ecological models of health behavior. Health Behavior and Health Education: Theory, Research, and Practice, 4, 465-486.

Saunders, T.J., Gray, C.E., Poitras, V.J., Chaput, J.P., Janssen, I., Katzmarzyk, P.T., Olds, T., Connor, S., Kho, M.E., Sampson, M., Tremblay, M.S., \& Carson, V. (2016). Combinations of physical activity, sedentary behaviour and sleep: Relationships with health indicators in schoolaged children and youth. Applied Physiology, Nutrition, and Metabolism, 41(6), 283-289.

Smith, A. L. (1999). Perceptions of peer relationships and physical activity participation in early adolescence. Journal of Sport \& Exercise Psychology, 21, 329-350.

Société canadienne de physiologie de l'exercice (2016). Directives canadiennes en matière de mouvement sur 24 heures pour les enfants et les jeunes (5 à 17 ans) : une approche intégrée regroupant l'activité physique, le comportement sédentaire et le sommeil. Repéré à https://csepguidelines.ca/fr/children-and-youth-5-17/ 
Stuntz, C.P. \& Weiss, M.R. (2010). Motivating children and adolescent to sustain a physically active lifestyle. American Journal of Lifestyle Medicine, 4(5), 433-444.

Thomas, J. \& Harden, A. (2008). Methods for the thematic synthesis of qualitative research in systematic reviews. BMC Medical Research Methodology, 8(45), 1-10. http://www.biomedcentral.com/1471-2288/8/45

Van den Berg, V., Vos, E. E., De Groot, R. H. M., Singh, A. S., \& Chinapaw, M. J. M. (2018). Untapped Resources: 10- to 13-Year-Old Primary Schoolchildren's Views on Additional Physical Activity in the School Setting: A Focus Group Study. International Journal of Environmental Research and Public Health, 15(12), 2713. https://doi.org/10.3390/ijerph15122713

Vors, O., Girard, A., Gal-Petitfaux, N., Lenzen, B., Mascret, N., Mouchet, A., Turcotte, S., \& Potdevin, F. (2020). A review of penetration of Francophone research on intervention in physical education and sport in Anglophone journals since 2010. Physical Education and Sport Pedagogy, 25(4), 331-345.

Wanless, E., Judge, L.W., Dieringer, S.T. \& Johnson, J.E. (2017). Recruiting Youth for AfterSchool Health Intervention Programming: Parent and Student Perceptions. Physical Educator, 74(1), 1-18.

Weiss, M.R. (2000). Motivating kids in physical activity. President's Council on Physical Fitness and Sport Research Digest. 3(11), 1-8. 